\title{
R. GLOWINSKI
}

\section{Sur l'approximation d'une inéquation variationnelle elliptique de type Bingham}

Revue française d'automatique, informatique, recherche opérationnelle. Analyse numérique, tome 10, no 3 (1976), p. 13-30.

$<$ http://www.numdam.org/item?id=M2AN_1976_10_3_13_0>

(C) AFCET, 1976, tous droits réservés.

L'accès aux archives de la revue "Revue française d'automatique, informatique, recherche opérationnelle. Analyse numérique » implique l'accord avec les conditions générales d'utilisation (http://www.numdam.org/legal. php). Toute utilisation commerciale ou impression systématique est constitutive d'une infraction pénale. Toute copie ou impression de ce fichier doit contenir la présente mention de copyright.

\section{Numdam}

Article numérisé dans le cadre du programme

Numérisation de documents anciens mathématiques

http://www.numdam.org/ 


\title{
SUR L'APPROXIMATION \\ D'UNE INÉQUATION VARIATIONNELLE ELLIPTIQUE DE TYPE BINGHAM
}

\author{
par R. GLowinski $\left({ }^{1}\right)$ \\ Communiqué par P.-G. Ciarlet
}

Résumé. - On étudie l'approximation par éléments finis d'ordre 1 d'une inéquation variationnelle elliptique, comportant un terme non différentiable, et modélisant des écoulements de fluides non newtoniens dans des conduites cylindriques

\section{INTRODUCTION}

Cet article est consacré à l'approximation, par éléments finis d'ordre 1, de l'inéquation variationnelle elliptique

$$
\left\{\mu \int_{\Omega} \nabla u \cdot \nabla(v-u) d x+g \int_{\Omega}|\nabla v| d x-g \int_{\Omega}|\nabla u| d x \geqq \int_{\Omega} f(v-u) d x,\right.
$$

Après avoir rappelé brièvement un certain nombre de résultats classiques sur (P) et son approximation on montre que l'estimation " presque évidente ", en $\sqrt{h}$, de l'erreur d'approximation dans $H_{0}^{1}(\Omega)$, établie dans GlowinskiLions-Trémolières [12] (chap. 5) n'est pas optimale puisqu'au paragraphe 5 on montre que dans certains cas importants dans les applications une estimation en $h \sqrt{-\log h}$ peut être obtenue.

\section{LE PROBLÈME CONTINU. MOTIVATION PHYSIQUE}

\subsection{Le problème continu}

On va reprendre succintement dans ce numéro un certain nombre de notions considérées et développées par ailleurs (par exemple dans Lions [13] (chap. 1), Cea-Glowinski [5], Duvaut-Lions [8] (chap. 6), Ekeland-Temam [9] (chap. 4), Glowinski-Lions-Trémolières [12] (chap. 1 et 5); cela permettra de fixer les notations et de rappeler certains résultats utiles dans la suite.

(1) Université Pierre-et-Marie-Curie, Laboratoire d'Analyse Numérique, Paris.

Revue Française d'Automatique, Informatique et Recherche Opérationnelle, décembre 1976. 
Soit $\Omega$ un ouvert borné de $\mathbf{R}^{2}$, de frontière $\Gamma$ régulière; on note $V$ l'espace d'Hilbert, de type Sobolev, $H_{0}^{1}(\Omega)$, soit

$$
H_{0}^{1}(\Omega)=\left\{v \mid v \in L^{2}(\Omega), \frac{\partial v}{\partial x_{1}} \in L^{2}(\Omega), i=1,2, v=0 \text { sur } \Gamma\right\} .
$$

On considère ensuite sur $V$ les formes et fonctionnelles $a, j, L$ définies par

$$
\begin{gathered}
a(u, v)=\int_{\Omega} \nabla u \cdot \nabla v d x . \\
j(v)=\int_{\Omega}|\nabla v| d x, \\
L(v)=\langle f, v\rangle, \quad f \in V^{\prime}=H^{-1}(\Omega) .
\end{gathered}
$$

Les quantités $\mu$ et $g$ étant deux constantes positives on considère l'inéquation variationnelle elliptique

$$
\left\{\begin{array}{c}
\mu a(u, v-u)+g j(v)-g j(u) \geqq L(v-u), \quad \forall v \in V, \\
u \in V .
\end{array}\right.
$$

REMARQUe $2.1: \mathrm{Si}$ on fait $g=0$ dans (P) on retrouve la formulation variationnelle du problème de Dirichlet

$$
-\mu \Delta u=f \text { dans } \Omega, \quad u=0 \quad \text { sur } \Gamma .
$$

L'ouvert $\Omega$ étant borné, la semi-norme $\left(\int_{\Omega}|\nabla v|^{2} d x\right)^{1 / 2}$ définit sur $V$ une norme - notée $\|v\|_{\mathrm{V}}$ dans la suite - équivalente à celle induite par $H^{1}(\Omega)$; il en résulte que $a$ est bilinéaire, continue et $V$-elliptique ( $a$ est également symétrique). Par ailleurs $j$ est convexe et continue sur $V$ et [inégalité de Schwarz dans $\left.L^{2}(\Omega)\right]$ :

$$
j(v-u) \leqq \sqrt{M(\Omega)}\|v-u\|_{V}
$$

en posant, dans (2.5), $M(\Omega)=$ aire de $\Omega$.

La forme $L$ étant continue sur $V$ il résulte des propriétés de $V, a, j$ que (P) admet une solution et une seule (on renvoie à l'un quelconque des travaux précités pour la démonstration de ce résultat).

On démontre par ailleurs dans H. Brézis [1] que si

$$
L(v)=\int_{\Omega} f v d x, \quad f \in L^{2}(\Omega)
$$

on a alors $u \in V \cap H^{2}(\Omega)$, avec, si $\Omega$ est convexe,

$$
\|u\|_{\mathrm{H}^{2}(\Omega)} \leqq \frac{\gamma(\Omega)}{\mu} \mid\|f\|_{L^{2}(\Omega)}
$$




\subsection{Motivation physique}

Si $L(v)=C \int_{\Omega} v d x(C>0$ par exemple), on démontre dans [8], (chap. 6) que (P) modélise l'écoulement laminaire stationnaire d'un fluide de Bingham dans une conduite cylindrique de section droite $\Omega, u(x)$ étant la vitesse de l'écoulement au point $x \in \Omega$ (on renvoie à Prager [18], Germain [11] et [8] (chap. 6)] pour la définition des fluides de Bingham). La constante $C$ représente la chute linéique de pression et $\mu$ et $g$ désignent, respectivement, la viscosité et le seuil de plasticité du fluide.

Le matériau ci-dessus se comporte comme un fluide visqueux (de viscosité $\mu$ ) dans

$$
\Omega^{+}=\{x|x \in \Omega,| \nabla u(x) \mid>0\}
$$

et comme un matériau rigide dans $\left({ }^{2}\right)$ :

$$
\Omega^{0}=\{x \mid x \in \Omega 2, \nabla u(x)=0\} ;
$$

on renvoie à Mossolov-Miasnikov $([14,15,16])$ pour, entre autres, une étude détaillée des propriétés de $\Omega^{+}$et $\Omega^{0}$.

\section{EXISTENCE DE MULTIPLICATEURS}

Soit

$$
\Lambda=\left\{q\left|q \in L^{2}(\Omega) \times L^{2}(\Omega),\right| q(x) \mid \leqq 1 \text { p.p. }\right\}
$$

avec $|q(x)|=\sqrt{q_{1}^{2}(x)+q_{2}^{2}(x)}$; on a alors le

THÉORÈME 3.1 : La solution u de (P) est caractérisée par l'existence de $p$ tel que

$$
\begin{gathered}
\mu a(u, v)+g \int_{\Omega} p . \nabla v d x=\langle f, v\rangle, \quad \forall v \in V, \\
\left.\begin{array}{c}
u \in V, \\
p . \nabla u=\underset{p \in \Lambda .}{|\nabla u| \text { p.p. }}
\end{array}\right\}
\end{gathered}
$$

Démonstration : On trouvera dans [5], [9] (chap. 4), [12] (chap. 1) des démonstrations du théorème ci-dessus basées sur le théorème de Hahn-Banach ou sur des théorèmes de min-max; dans ce qui suit on va reprendre une démonstration "plus constructive ", donnée dans [12] (chap. 5), et basée sur un procédé de régularisation.

La démonstration du fait que $\{u, p\}$ solution de (3.1) et (3.2) implique $u$ solution de $(\mathrm{P})$ est à peu près immédiate.

${ }^{2}$ ) On notera le caractère de problème à frontière libre de (P).

décembre 1976. 
Faisant $v=0$ et $v=2 u$ dans (P) on en déduit par addition

$$
\mu a(u, u)+g j(u)=\langle f, u\rangle .
$$

Soit $\varepsilon>0$, on régularise $j$ par $j_{\varepsilon}$ définie par

$$
j_{\varepsilon}(v)=\int_{\Omega} \sqrt{\varepsilon^{2}+|\nabla v|^{2}} d x ;
$$

la fonctionnelle $j_{\varepsilon}$ étant convexe et continue sur $V$ le problème régularisé

$$
\left\{\begin{array}{c}
\mu a\left(u_{\varepsilon}, v-u_{\varepsilon}\right)+g j_{\varepsilon}(v)-g j_{\varepsilon}\left(u_{\varepsilon}\right) \geqq L\left(v-u_{\varepsilon}\right), \quad \forall v \in V, \\
u_{\varepsilon} \in V,
\end{array}\right.
$$

admet une solution et une seule.

On va montrer que $\lim _{\varepsilon \rightarrow 0} u_{\varepsilon}=u$ dans $V$ fort; on a d'après $(\mathrm{P})$ ett $_{q}\left(\mathrm{P}_{\varepsilon}\right)$ :

$$
\begin{aligned}
& \mu a\left(u, u_{\varepsilon}-u\right)+g j\left(u_{\varepsilon}\right)-g j(u) \geqq L\left(u_{\varepsilon}-u\right), \\
& \mu a\left(u_{\varepsilon}, u-u_{\varepsilon}\right)+g j_{\varepsilon}(u)-g j_{\varepsilon}\left(u_{\varepsilon}\right) \geqq L\left(u-u_{\varepsilon}\right),
\end{aligned}
$$

d'où par addition :

$$
\mu a\left(u_{\varepsilon}-u, u_{\varepsilon}-u\right)+g\left(j_{\varepsilon}\left(u_{\varepsilon}\right)-j\left(u_{\varepsilon}\right)\right) \leqq g\left(j_{\varepsilon}(u)-j(u)\right) .
$$

Par ailleurs

implique

$$
0<\sqrt{t^{2}+\varepsilon^{2}}-|t|=\frac{\varepsilon^{2}}{\sqrt{t^{2}+\varepsilon^{2}}+|t|} \leqq \varepsilon, \quad \forall t \in \mathbf{R}
$$

$$
0 \leqq j_{\varepsilon}(v)-i(v) \leqq \varepsilon M(\Omega), \quad \forall v \in V
$$

d'où, avec (3.4),

soit encore

$$
\mu a\left(u_{\varepsilon}-u, u_{\varepsilon}-u\right) \leqq g \varepsilon M(\Omega),
$$

$$
\text { i } u_{\varepsilon}-u \|_{V} \leqq \sqrt{M(\Omega)}\left(\begin{array}{l}
g \\
\varepsilon
\end{array}\right)^{1 / 2}
$$

On a donc démontré

$$
\lim _{\varepsilon \rightarrow 0} u_{\varepsilon}=u \text { dans } V \text { fort. }
$$

La fonctionnelle $j_{\varepsilon}$ étant différentiable sur $V,\left(\mathrm{P}_{\mathrm{\varepsilon}}\right)$ équivaut à l'équation variationnelle non linéaire

$$
\left.\mu a\left(u_{\varepsilon}, v\right)+g\left\langle j_{\varepsilon}^{\prime}\left(u_{\varepsilon}\right), v\right\rangle=L(v), \quad \forall v \in V^{\prime},\right\}
$$

Revue Française d'Autornatiqüe, İ̀nformatique et Recherche Opérationnelle 
avec

$$
\left\langle j_{\varepsilon}^{\prime}(w), v\right\rangle=\int_{\Omega} \frac{\nabla w \cdot \nabla v}{\sqrt{\varepsilon^{2}+|\bar{v}|^{2}}} d x, \quad \forall v, w \in V
$$

on pose

$$
p_{\varepsilon}=\frac{\nabla u_{\varepsilon}}{\sqrt{\varepsilon^{2}+\left|\nabla u_{\varepsilon}\right|^{2}}}
$$

On a alors

$$
p_{\varepsilon} \in \Lambda
$$

et, compte tenu de (3.7)-(3.9),

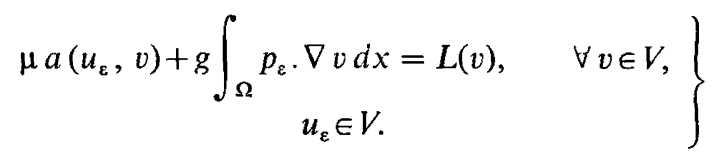

Le convexe $\dot{\Lambda}$ étant borné et fermé dans $L^{2}(\Omega) \times L^{2}(\Omega)$ on peut extraire de $\left(p_{\varepsilon}\right)_{\varepsilon}$ une sous-suite, encore notée $\left(p_{\varepsilon}\right)_{\varepsilon}$, telle que

$$
\left.\begin{array}{c}
\lim _{\varepsilon \rightarrow 0} p_{\varepsilon}=p \text { dans } L^{2}(\Omega) \times L^{2}(\Omega) \text { faible }\left({ }^{3}\right), \\
p \in \Lambda .
\end{array}\right\}
$$

A la limite dans (3.11) on a, compte tenu de (3.6) et (3.12),

$$
\left.\mu a(u, v)+g \int_{\Omega} p \cdot \nabla v d x=L(v), \quad \forall v \in V,\right\}
$$

soit (3.1).

Pour démontrer complètement le théorème il reste à montrer

$$
p . \nabla u=|\nabla u| \mathrm{p} . \mathrm{p} .
$$

on prend $v=u$ dans (3.13), d'où par comparaison avec (3.3) :

$$
\int_{\Omega}|\nabla u| d x-\int_{\Omega} p \cdot V u d x=\int_{\Omega}(|\nabla u|-p \cdot \nabla u) d x=0 .
$$

On a $p \in \Lambda$, d'où (inégalité de Schwarz dans $\mathbf{R}^{2}$ ) :

$$
p . \nabla u \leqq|\nabla u| \text { p.p. }
$$

ce qui, avec (3.15), implique (3.14).

$\left(^{3}\right)$ On a, en fait $p_{\varepsilon} \rightarrow p$ dans $L^{\infty}(\Omega) \times L^{\infty}(\Omega)$ faible *. décembre 1976. 
RemarQue $3.1:$ La fonction $p \in \Lambda$ qui vient d'être mise en évidence au théorème 3.1 n'est pas unique en général comme le montre le contre-exemple donné en [12] (chap. 5).

ReMARQUe 3.2 : On montrerait facilement que le couple $\{u, p\}$ solution de (3.1) et (3.2) est point-selle sur $V \times \Lambda$ de la fonctionnelle $\mathscr{L}$ définie par

$$
\mathscr{L}(v, q)=\frac{\mu}{2} \int_{\Omega}|\nabla v|^{2} d x+g \int_{\Omega} q \cdot \nabla v d x-\langle f, v\rangle .
$$

Remarque 3.3 : On peut déduire de l'existence de $\{u, p\}$, solution de (3.1) et (3.2), des méthodes itératives (de type Uzawa, cf. [12], chap. 2) permettant de résoudre efficacement et simplement le problème $(\mathrm{P})$ par réduction à une suite de problèmes de Dirichlet homogènes pour $-\Delta$; on renvoie à [5], [12] (chap. 5), Fortin [10] et Bristeau [3] pour l'étude et des exemples d'application de ces méthodes à la résolution numérique de $(\mathrm{P})$.

\section{APPROXIMATION PAR ÉLÉMENTS FINIS D'ORDRE 1}

Dans ce numéro on suit [12] (chap. 5); par ailleurs, pour simplifier l'exposé on supposera que $\Omega$ est un domaine polygonal de $\mathbf{R}^{2}$.

\subsection{Définition du problème approché}

Soit $\mathscr{T}_{h}$ une triangulation finie de $\Omega$ vérifiant

$$
\begin{gathered}
T \subset \bar{\Omega} \forall T \subset \mathscr{T}_{h}, \quad \bigcup_{T \in \mathscr{F}_{h}} T=\bar{\Omega}, \\
\dot{T} \cap \dot{T}^{\prime}=\varnothing, \quad \forall T, T^{\prime} \in \mathscr{T}_{h}, \quad T \neq T^{\prime}, \\
T, T^{\prime} \in \mathscr{T}_{h}, T \neq T^{\prime \prime} \rightarrow T \cap T^{\prime}=\varnothing,
\end{gathered}
$$

ou $T$ et $T^{\prime}$ n'ont qu'un sommet commun ou ont tout un côté commun. $\}$

On prend $h$ égal à la longueur du plus grand côté des $T \in \mathscr{T}_{h}$ et on approche $V$ par

$$
V_{h}=\left\{v_{h} \mid v_{h} \in C^{0}(\bar{\Omega}), v_{h}=0 \text { sur } \Gamma,\left.v_{h}\right|_{T} \in P_{1}, \forall T \in \mathscr{T}_{h}\right\}
$$

avec $P_{1}=$ espace des polygônes de degré $\leqq 1$; on a $V_{h} \subset V$, ce qui permet d'approcher - formellement pour l'instant - (P) par le problème en dimension finie.

$$
\left\{\begin{array}{c}
\mu a\left(u_{h}, v_{h}-u_{h}\right)+g j\left(v_{h}\right)-g j\left(u_{h}\right) \geqq\left\langle f, v_{h}-u_{h}\right\rangle, \quad \forall v_{h} \in V_{h}, \\
u_{h} \in V_{h} .
\end{array}\right.
$$

$V_{h}$ étant un sous-espace fermé de $V$, on a la

Proposition 4.1 : Le problème approché $\left(\mathrm{P}_{h}\right)$ admet une solution et une seule. 
ReMARQue 4.1 : On s'est limité dans ce travail à une approximation par éléments finis d'ordre 1, cela étant justifié par l'existence d'un seuil de régularité pour les solutions de (P), qui fait qu'en général, et même avec des données très régulières on a $u \notin V \cap H^{3}(\Omega)$ ( $c f$. l'exemple du paragraphe 5). Cela étant on trouvera dans [10], [3], [12] (chap. 5), Bristeau-Glowinski [4] des travaux sur l'approximation de $(\mathrm{P})$ par des éléments finis d'ordre 2, droits et isoparamétriques, qui semblent montrer qu'à nombre de degrés de liberté égaux les précisions obtenues avec les éléments finis d'ordre 1 et 2 sont comparables mais que les éléments d'ordre 2 sont beaucoup plus coûteux à mettre en cuvre (occupation mémoire, temps de calcul, etc.). Notons également que dans la mise en œuvre des éléments d'ordre 1, le calcul de $\int_{\Omega}|\nabla v| d x$ peut se faire exactement alors que les éléments d'ordre 2 nécessitent l'emploi d'une méthode d'intégration numérique.

REMARQUe 4.2 : Le seuil de régularité mentionné ci-dessus semble être un phénomène assez général pour les problèmes non linéaires.

\subsection{Convergence des solutions approchées (cas général)}

\section{On a le}

THÉORÈME $4.1:$ Si, lorsque $h \rightarrow 0$, les angles de $\mathscr{T}_{h}$ sont bornés inférieurement, uniformément en $h$, par $\theta_{0}>0$, on $a$

$$
\lim _{h \rightarrow 0}\left\|u_{h}-u\right\|_{V}=0
$$

$u$ et $u_{h}$ étant les solutions respectives de $(\mathrm{P})$ et $\left(\mathrm{P}_{h}\right)$.

Démonstration: On suit [12] (chap. 5); faisant $v_{h}=0$ dans $\left(\mathbf{P}_{h}\right)$ on en dédüit :

$$
\left\|u_{h}\right\|_{V} \leqq \frac{1}{\mu}\|f\|_{*}, \quad \forall h,
$$

avec

$$
\|f\|_{*}=\sup _{\substack{v \neq 0 \\ v \in V}} \frac{|\langle f, v\rangle|}{\|v\|_{V}} .
$$

On a par ailleurs

$$
\begin{aligned}
& \mu a\left(u_{h}, v_{h}-u_{h}\right)+g j\left(v_{h}\right)-g j\left(u_{h}\right) \geqq\left\langle f, v_{h}-u_{h}\right\rangle, \quad \forall v_{h} \in V_{h}, \\
& \mu a\left(u, u_{h}-u\right)+g j\left(u_{h}\right)-g j(u) \geqq\left\langle f, u_{h}-u\right\rangle,
\end{aligned}
$$

d'où par addition

$$
\begin{gathered}
\mu a\left(u_{h}-u, u_{h}-u\right) \leqq g j\left(v_{h}\right)-\underset{j}{g}(u)+\mu a\left(u_{h}, v_{h}-u\right)-\left\langle f, v_{h}-u\right\rangle, \\
\forall v_{h} \in V_{h} .
\end{gathered}
$$


Compte tenu de (2.5) et (4.6) on déduit de (4.7) :

$$
\left\|u_{h}-u\right\|_{V}^{2} \leqq \frac{1}{\mu}\left(g \sqrt{M(\Omega)}+2\|f\|_{*}\right)\left\|v_{h}-u\right\|_{\mid V}, \quad \forall v_{h} \in V_{h} .
$$

Soit $\varphi \in \mathscr{D}(\Omega)$, on note $r_{h} \varphi$ l'interpolé « linéaire " de $\varphi$ sur $\mathscr{T}_{h}$, soit

$$
\left\{\begin{array}{c}
r_{h} \varphi \in V_{h}, \\
r_{h} \varphi(P)=\varphi(P), \quad \forall P \text { sommet de } \mathscr{T}_{h} .
\end{array}\right.
$$

Sous les hypothèses faites sur $\mathscr{T}_{h}$ dans l'énoncé du théorème 4.1 , on a (cf., par exemple, Strang-Fix [19], Ciarlet-Raviart [6]) :

$$
\left\|r_{h} \varphi-\varphi\right\|_{V} \leqq \gamma\|\varphi\|_{W^{2}, \infty(\Omega)} h, \quad \forall \varphi \in \mathscr{D}(\Omega)
$$

avec $\gamma$ indépendant de $h$ et $\varphi$.

De (4.8), (4.9) et de l'inégalité du triangle on déduit

$$
\left.\begin{array}{c}
\left\|u_{h}-u\right\|_{V}^{2} \leqq \frac{1}{\mu}\left(g \sqrt{M(\Omega)}+2\|f\|_{*}\right)\left(\|\varphi-u\|_{V}+\gamma\|\varphi\|_{W^{2, \infty}(\Omega)} h\right), \\
\forall \varphi \in \mathscr{D}(\Omega),
\end{array}\right\}
$$

ce qui, avec la densité de $\mathscr{D}(\Omega)$ dans $V$, implique (4.5).

\subsection{Convergence des solutions approchées $f \in L^{2}(\Omega)$}

Comme déjà signalé au paragraphe 2.1 il résulte de [1] que si la frontière $\Gamma$ est assez régulière, alors $f \in L^{2}(\Omega)$ implique $u \in V \cap H^{2}(\Omega)$ avec, si $\Omega$ est convexe,

$$
\|u\|_{H^{2}(\Omega)} \leqq \frac{\gamma_{0}(\Omega)}{\mu}\|f\|_{L^{2}(\Omega)} .
$$

Les résultats ci-dessus sont donc, en particulier, vrais si $\Omega$ est un polygone convexe, ce que l'on supposera dans ce qui suit; dans ces conditions on a le

THÉORÈME 4.2 : Les hypothèses sur $\mathscr{T}_{h}$ étant celles du théorème 4.1 on $a$, si $f \in L^{2}(\Omega)$ :

$$
\left\|u_{h}-u\right\|_{V}=O(\sqrt{ } h)
$$

Démonstration : La relation (4.7), l'inégalité de Schwarz dans $V$ et $u \in H_{0}^{1}(\Omega) \cap H^{2}(\Omega)$ impliquent successivement, $\forall v_{h} \in V_{h}$,

$$
\begin{aligned}
\mu\left\|u_{h}-u\right\|_{V}^{2} & \leqq \mu a\left(u_{h}-u, v_{h}-u\right)+g j\left(v_{h}-u\right)+\mu a\left(u, v_{h}-u\right)-\int_{\Omega} f\left(v_{h}-u\right) d v, \\
\frac{\mu}{2}\left\|u_{h}-u\right\|_{V}^{2} & \leqq \frac{\mu}{2}\left\|v_{h}-u\right\|_{V}^{2}+g j\left(v_{h}-u\right)+\mu \int_{\Omega} \nabla u \cdot \nabla\left(v_{h}-u\right) d x-\int_{\Omega} f\left(v_{h}-u\right) d x, \\
& \leqq \frac{\mu}{2}\left\|v_{h}-u\right\|_{V}^{2}+g j\left(v_{h}-u\right)+\int_{\Omega}(-\mu \Delta u-f)\left(v_{h}-u\right) d x,
\end{aligned}
$$

Revue Française d'Automatique, Informatique et Recherche Opírationnelle' 
d'où

$$
\left.\begin{array}{rl}
\frac{\mu}{2}\left\|u_{h}-u\right\|_{V}^{2} \leqq & \frac{\mu}{2}\left\|v_{h}-u\right\|_{V}^{2}+g j\left(v_{h}-u\right) \\
& +\left(\mu\|\Delta u\|_{L^{2}(\Omega)}+\|f\|_{L^{2}(\Omega)}\right)\left\|v_{h}-u\right\|_{L^{2}(\Omega)}, \\
\forall v_{h} \in V_{h} .
\end{array}\right\}
$$

L'application $v \rightarrow\|\Delta v\|_{L^{2}(\Omega)}$ définissant sur $V \cap H^{2}(\Omega)$ une norme équivalente à celle induite par $H^{2}(\Omega)$, on déduit de (2.5), (4.11) et (4.13) :

$$
\left.\begin{array}{c}
\frac{\mu}{2}\left\|u_{h}-u\right\|_{V}^{2} \leqq \\
\frac{\mu}{2}\left\|v_{h}-u\right\|_{V}^{2}+g \sqrt{M(\Omega)}\left\|v_{h}-u\right\|_{V} \\
+\left(1+\gamma_{0}(\Omega)\right)\|f\|_{L^{2}(\Omega)}\left\|v_{h}-u\right\|_{L^{2}(\Omega)}, \\
\forall v_{h} \in V_{h} .
\end{array}\right\}
$$

On a $\Omega \subset \mathbf{R}^{2}$ avec $\Gamma$ polygonal, donc lipschitzien; cela implique ( $c f$. Necas [17]) que $H^{2}(\Omega) \subset C^{0}(\Omega)$ avec injection continue.

La solution $u$ de $(P)$ vérifiant $u \in H^{2}(\Omega)$ on peut alors définir l'interpolé linéaire $r_{h} u$ de $u$, soit

$$
\left\{\begin{array}{l}
r_{h} u \in V_{h}, \\
r_{h} u(P)=u(P) \quad \text { pour tout sommet } P \text { de } \mathscr{T}_{h} ;
\end{array}\right.
$$

sous les hypothèses faites sur $\mathscr{T}_{h}$ on a (cf. [19] et [6]) :

$$
\begin{aligned}
& \left\|r_{h} u-u\right\|_{V} \leqq \gamma_{1} h\|u\|_{H^{2}(\Omega)}, \\
& \left\|r_{h} u-u\right\|_{L^{2}(\Omega)} \leqq \gamma_{2} h^{2}\|u\|_{H^{2}(\Omega)},
\end{aligned}
$$

avec, dans (4.15) et (4.16), $\gamma_{1}$ et $\gamma_{2}$ indépendants de $u$ et $h$.

Faisant alors $v_{h}=r_{h} u$ dans (4.14) on déduit de (4.11), (4.15) et (4.16) :

$$
\begin{aligned}
\frac{\mu}{2}\left\|u_{h}-u\right\|_{V}^{2} \leqq & \frac{\gamma_{0}\|f\|_{L^{2}(\Omega)}}{\mu} \\
& \times\left[\left(\frac{\gamma_{1}^{2} \gamma_{0}}{2}+\left(1+\gamma_{0}\right) \gamma_{2}\right)\|f\|_{L^{2}(\Omega)} h^{2}+g \sqrt{M}(\Omega) \gamma_{1} h\right]
\end{aligned}
$$

en posant $\gamma_{0}=\gamma_{0}(\Omega)$. La relation (4.17) démontre - et précise - (4.12).

REMARQUe 4.3 : L'estimation (4.12) n'est pas nécessairement optimale comme on le verra au paragraphe 5 .

REMARQUE 4.4 : On pourrait déduire (4.12) plus rapidement en utilisant la relation (4.8), mais (4.17) précise (4.12), et en particulier redonne, pour $g=0$, le $O(h)$ du cas linéaire.

décembre 1976. 
5. CAS DU DOMAINE CiRCulaIRe AVEC $f=$ Cte. GÉNERAlisation

Dans ce numéro on particularise $(\mathrm{P})$ au cas où

$$
\begin{gathered}
\Omega=\left\{x \mid x \in \mathbf{R}^{2}, \sqrt{x_{1}^{2}+x_{2}^{2}}<R\right\}, \\
L(v)=C \int_{\Omega} v d x, \quad C>0 .
\end{gathered}
$$

\subsection{Solution exacte, propriétés de régularité}

Sous les conditions (5.1) et (5.2) la solution de (P) est connue exactement et est donnée, en posant $r=\sqrt{x_{1}^{2+} x_{2}^{2}}$, par

$$
u(x) \equiv 0 \quad \text { si } \quad g \geqq \frac{C R}{2},
$$

et si $g<C R / 2$ par

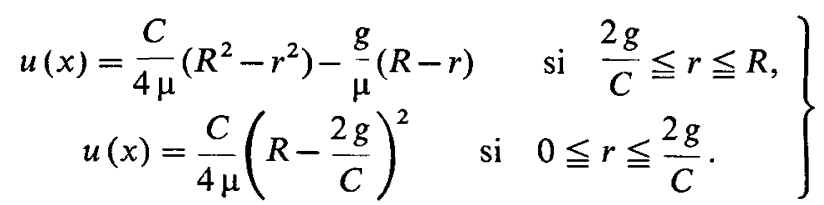

On supposera dans ce qui suit que $g<C R / 2$; il résulte alors de (5.4) que

$$
\left.\begin{array}{l}
u \in V \cap W^{2, \infty}(\Omega), \\
u \notin V \cap H^{3}(\Omega),
\end{array}\right\}
$$

ce qui complète la remarque 4.1 .

\subsection{Approximation par éléments finis d'ordre 1}

Soit $\mathscr{T}_{h}$ une triangulation finie de $\Omega$ vérifiant (4.2), (4.3) et

$$
\forall T \in \mathscr{T}_{h}, \quad T \subset \Omega ;
$$

soit $\Omega_{h}=\overbrace{T \in \mathscr{T}_{h}} T$ et $\Gamma_{h}=\partial \Omega_{h}$, on a alors $\Omega_{h} \subset \Omega$ et dans ce qui suit on supposera que $\Gamma_{h} \in \mathcal{F}_{h}$ vérifie :

tous les sommets de $\Gamma_{h}$ sont situés sur $\Gamma$.

On approche alors $V$ par

$$
V_{h}=\left\{v_{h} \mid v_{h} \in C^{0}\left(\bar{\Omega}_{h}\right), v_{h}=0 \text { sur } \Gamma_{h},\left.v_{h}\right|_{T} \in P_{1} \forall T \in \mathscr{T}_{h}\right\} ;
$$

$V_{h}$ peut être considéré comme un sous-espace de $V$, obtenu en prolongeant 
$v_{h} \in V_{h}$ par zéro dans $\Omega-\Omega_{h}$. Dans ces conditions il est licite d'approcher (P) par

$\left(\mathrm{P}_{h}\right) \quad\left\{\begin{array}{c}\mu a\left(u_{h}, v_{h}-u_{h}\right)+g j\left(v_{h}\right)-g j\left(u_{h}\right) \geqq C \int_{\Omega}\left(v_{h}-u_{h}\right) d x, \quad \forall v_{h} \in V_{h}, \\ u_{h} \in V_{h},\end{array}\right.$

problème en dimension finie qui admet une solution et une seule.

5.3. Une estimation de l'erreur d'approximation en $h \sqrt{-\log h}$

L'estimation de l'erreur ci-après va résulter essentiellement des trois lemmes ci-dessous :

Lemme 5.1 : Soit $\vec{p}, \vec{q} \in \mathbf{R}^{2}, \vec{p}$ et $\vec{q} \neq 0$, alors

$$
\left|\frac{\vec{p}}{|\vec{p}|}-\frac{\vec{q}}{|\vec{q}|}\right| \leqq 2 \frac{|\vec{p}-\vec{q}|}{|\vec{p}|+|\vec{q}|}
$$

Démonstration : On a

or

$$
(|\vec{p}|+|\vec{q}|)\left(\frac{\vec{p}}{|\vec{p}|}-\frac{\vec{q}}{|\vec{q}|}\right)=(\vec{p}-\vec{q})+\left(\frac{|\vec{q}|}{|\vec{p}|} \vec{p}-\frac{|\vec{p}|}{|\vec{q}|} \vec{q}\right)
$$

d'où

$$
\left|\frac{|\vec{q}|}{|\vec{p}|} \vec{p}-\frac{|\vec{p}|}{|\vec{q}|} \vec{q}\right|^{2}=\vec{p}^{2}+\vec{q}^{2}-2 \vec{p} \cdot \vec{q}=|\vec{p}-\vec{q}|^{2},
$$

$$
(|\vec{p}|+|\vec{q}|)\left|\frac{\vec{p}}{|\vec{p}|}-\frac{\vec{q}}{|\vec{q}|}\right| \leqq 2|\vec{p}-\vec{q}|
$$

C. Q. F. D.

RemarQue 5.1 : Dans (5.8), 2 est la meilleure constante possible (prendre $\vec{p}=-\vec{q}$ ); par ailleurs (5.8) vaut également dans $\mathbf{R}^{\mathrm{N}}, N \geqq 3$.

LEMME $5.2:$ Soit $u$ et $u_{h}$ les solutions respectives de $(P)$ et $\left(P_{h}\right)$ et $p$ vérifiant (3.1) et (3.2); on a alors

$$
\left.\begin{array}{c}
\mu a\left(u_{h}-u, u_{h}-u\right) \leqq \mu a\left(u_{h}-u, v_{h}-u\right)+g \int_{\Omega}\left(p_{h}-p\right) \cdot \nabla\left(v_{h}-u\right) d x, \\
\text { pour tout } p_{h} \in \Lambda . \text { tel que } p_{h} . \nabla v_{h}=\left|\nabla v_{h}\right| \text { p.p., } \quad \forall v_{h} \in V_{h} .
\end{array}\right\}
$$

Démonstration : On va démontrer (5.9) dans le cas général i. e. avec $f \in V^{\prime}$. On part de (4.7) soit $\mu a\left(u_{h}-u, u_{h}-u\right) \leqq \mu a\left(u_{h}, v_{h}-u\right)+g j\left(v_{h}\right)-g j(u)-\left\langle f, v_{h}-u\right\rangle, \quad \forall v_{h} \in V_{h} ;$ décembre 1976. 
on en déduit

$$
\begin{aligned}
\mu a\left(u_{h}-u, u_{h}-u\right) \leqq & \mu a\left(u_{h}-u, v_{h}-u\right) \\
& +g j\left(v_{h}\right)-g j(u)+\mu a\left(u, v_{h}-u\right)-\left\langle f, v_{h}-u\right\rangle, \quad \forall v_{h} \in V_{h},
\end{aligned}
$$

d'où, compte tenu de (3.1),

$$
\begin{aligned}
\mu a\left(u_{h}-u, u_{h}-u\right) \leqq & \mu a\left(u_{h}-u, v_{h}-u\right) \\
+ & g j\left(v_{h}\right)-g j(u)-g \int_{\Omega} p \cdot \nabla\left(v_{h}-u\right) d x, \\
& \vee v_{h} \in V_{h} .
\end{aligned}
$$

Pour tout $v_{h} \in V_{h}$, l'ensemble des $p_{h} \in \Lambda$ tels que

$$
p_{h} \cdot \nabla v_{h}=\left|\nabla v_{h}\right| \text { p. p. }
$$

est non vide; on a alors

$$
\begin{gathered}
j\left(v_{h}\right)=\int_{\Omega} p_{h} \cdot \nabla v_{h} d x, \\
j(u)=\int_{\Omega}|\nabla u| d x \geqq \int_{\Omega} p_{h} . \nabla u d x,
\end{gathered}
$$

ce qui implique, avec (5.10), l'inégalité (5.9) à démontrer.

Soit $u$ la solution de $(P)$, et $\delta>0$; on définit $\Omega^{\delta} \subset \Omega$ par

$$
\Omega^{\delta}=\{x|x \in \Omega,| \nabla u(x) \mid>\delta\} ;
$$

dans le cas du problème $(P)$, associé à (5.1) et (5.2), on a (en supposant $g<C R / 2)$ le

LEMME $5.3:$ On $a$

$$
\begin{aligned}
\int_{\Omega^{\delta} \delta} \frac{d x}{|\nabla u|}=\frac{4 \pi \mu}{C}[- & \frac{2 \mu}{C} \delta+\left(R-\frac{2 g}{C}\right) \\
& \left.+\frac{2 g}{C} \log \left(R-\frac{2 g}{C}\right)-\frac{2 g}{C} \log \frac{2 \mu}{C} \delta\right] .
\end{aligned}
$$

Démonstration: Compte tenu de (5.4) on a

d'où

$$
\left|\frac{d u}{d r}\right|=\frac{1}{\mu}\left(\frac{C r}{2}-g\right) \quad \text { si } \quad \frac{2 g}{C} \leqq r \leqq R,
$$

$$
\int_{\Omega^{\delta}} \frac{d x}{|\nabla u|}=2 \pi \mu \int_{2 / C(\mu \delta+g)}^{R} \frac{r d r}{(C r / 2)-g},
$$

ce qui implique (5.13). 
Des résultats ci-dessus on va déduire le

THÉORÈme 5.1: Soit u la solution du problème $(P)$ associé à (5.1) et (5.2), $u_{h}$ celle du problème $\left(P_{h}\right)$ correspondant, avec $\mathscr{T}_{h}$ vérifiant $(5.7)$; si lorsque $h \rightarrow 0$ les angles de $\mathscr{T}_{h}$ sont bornés inférieurement, uniformément en $h$, par $\theta_{0}>0$, on $a$

$$
\left\|u_{h}-u\right\|_{v}=O(h \sqrt{-\log h}) .
$$

Démonstration : On part du lemme 5.2; de (5.9) on déduit

$$
\left.\begin{array}{l}
\frac{\mu}{2}\left\|u_{h}-u\right\|_{V}^{2} \leqq \frac{\mu}{2}\left\|r_{h} u-u\right\|_{V}^{2}+g \int_{\Omega}\left|p_{h}-p\right| \cdot\left|\nabla\left(r_{h} u-u\right)\right| d x, \\
\quad \forall p_{h} \in \Lambda \\
\text { tel que } \\
\quad \nabla r_{h} u=\left|\nabla r_{h} u\right|,
\end{array}\right\}
$$

avec, dans (5.15), $r_{h} u$ défini par

$$
\left\{\begin{array}{c}
r_{h} u \in V_{h}, \\
r_{h} u(P)=u(P), \quad \forall P \text { sommet de } \mathscr{T}_{h} .
\end{array}\right.
$$

On a $r_{h} u=0 \operatorname{sur} \Omega-\Omega_{h}$, d'où

Posons

$$
\begin{aligned}
\left\|r_{h} u-u\right\|_{V}^{2}= & \int_{\Omega}\left|\nabla\left(r_{h} u-u\right)\right|^{2} d x= \\
& \int_{\Omega-\Omega_{h}}|\nabla u|^{2} d x+\int_{\Omega_{h}}\left|\nabla\left(r_{h} u-u\right)\right|^{2} d x .
\end{aligned}
$$

$$
X_{1}=\frac{\mu}{2} \int_{\Omega-\Omega_{h}}|\nabla u|^{2} d x, \quad X_{2}=\frac{\mu}{2} \int_{\Omega_{h}}\left|\nabla\left(r_{h} u-u\right)\right|^{2} d x
$$

On montrerait aisément

$$
\operatorname{Aire}\left(\Omega-\Omega_{h}\right)<\frac{\pi}{4} h^{2},
$$

par ailleurs (5.4) implique

$$
|\nabla u(x)| \leqq \frac{C}{2 \mu}\left(R-\frac{2 g}{C}\right), \quad \forall x \in \Omega,
$$

d'où, avec (5.17),

$$
X_{1} \leqq \frac{\pi}{32 \mu} C^{2}\left(R-\frac{2 g}{C}\right)^{2} h^{2}
$$

On a $u \in W^{2, \infty}(\Omega)$, d'où sur chaque triangle $T \in \mathscr{T}_{h}\left(c f_{.}\right.$, par exemple, Ciarlet-Wagschal [7]) :

$$
\left|\nabla\left(r_{h} u-u\right)(x)\right| \leqq \frac{2 h}{\sin \theta_{0}}\left\|\rho\left(D_{2} u\right)\right\|_{L^{\infty}(T)},
$$

décembre 1976. 
où dans (5.20), $D_{2} u(x)$ désigne la matrice hessienne de $u$ au point $x$, soit

$$
D_{2} u(x)=\left(\begin{array}{ll}
\frac{\partial^{2} u}{\partial x_{1}^{2}}(x) & \frac{\partial^{2} u}{\partial x_{1} \partial x_{2}}(x) \\
\frac{\partial^{2} u}{\partial x_{1} \partial x_{2}}(x) & \frac{\partial^{2} u}{\partial x_{2}^{2}}(x)
\end{array}\right),
$$

et $\rho\left(D_{2} u(x)\right)$ le rayon spectral de $D_{2} u(x)$. On a

$$
D_{2} u(x)=0 \quad \text { si } 0 \leqq r<\frac{2 g}{C}, \quad \text { d'où } \quad \rho\left(D_{2} u(x)\right)=0,
$$

et on montrerait que

$$
\rho\left(D_{2} u(x)\right)=\frac{C}{2 \mu} \quad \text { si } \quad \frac{2 g}{C}<r<R ;
$$

(5.20)-(5.22) impliquent alors

$$
X_{2} \leqq \frac{\pi}{\mu} R\left(R-\frac{2 g}{C}+h\right) C^{2}\left(\frac{h}{\sin \theta_{0}}\right)^{2} .
$$

Il reste à estimer, dans (5.15), le terme

On a

$$
g \int_{\Omega}\left|p_{h}-p\right| \cdot\left|\nabla\left(n_{h} u-u\right)\right| d x
$$

avec

$$
\bar{\Omega}=\bigcup_{i=3}^{6} \bar{\Omega}_{i}
$$

$$
\begin{aligned}
& \Omega_{3}=\Omega_{-} \Omega_{h}, \\
& \Omega_{4}=\left\{x \mid x \in \Omega_{h}, r>\frac{2 g}{C}+h\right\}, \\
& \Omega_{5}=\left\{x \mid x \in \Omega_{h}, \frac{2 g}{C}-h<r<\frac{2 g}{C}+h\right\}, \\
& \Omega_{6}=\left\{x \mid x \in \Omega_{h}, 0 \leqq r<\frac{2 g}{C}-h\right\} ;
\end{aligned}
$$

posons, pour $3 \leqq i \leqq 6$,

$$
X_{i}=g \int_{\Omega_{i}}\left|p_{h}-p\right| \cdot\left|\nabla\left(r_{h} u-u\right)\right| d x .
$$

Sur $\Omega-\Omega_{h}$ on a $r_{h} u=0$, on peut donc prendre, dans (5.15),

$$
p_{h}=0 \text { sur } \Omega-\Omega_{h} ;
$$


de (5.17), (5.18), (5.26), et de $p \in \Lambda$, on déduit alors

$$
X_{3} \leqq g \int_{\Omega-\Omega_{h}}|\nabla u| d x \leqq \frac{\pi}{8 \mu} g C\left(R-\frac{2 g}{C}\right) h^{2} .
$$

De (5.20)-(5.22) et de $p, p_{h} \in \Lambda$, on déduit

$$
X_{5} \leqq 2 g \frac{C}{\mu} \text { Aire }\left(\Omega_{5}\right) \frac{h}{\sin \theta_{0}},
$$

d'où

$$
X_{5} \leqq \frac{16 \pi}{\mu} g^{2} \frac{h^{2}}{\sin \theta_{0}} .
$$

Par définition de $h$ on a $r_{h} u=u=$ Cte sur $\Omega_{6}$, d'où

$$
X_{6}=0 \text {. }
$$

Il reste donc à évaluer $X_{4}$; les équipotentielles de $u$ dans $\Omega_{4}$ étant des cercles, pour $h$ suffisamment petit on a

d'où

$$
\left.\begin{array}{lll}
\left.r_{h} u\right|_{T} \neq \text { Cte }, & \forall T \in \mathscr{T}_{h}, & T \subset \Omega_{4}, \\
\left.\nabla r_{h} u\right|_{T} \neq 0 & \forall T \in \mathscr{T}_{h}, & T \subset \bar{\Omega}_{4} .
\end{array}\right\}
$$

Compte tenu de (5.30) on a donc, dans (5.15) :

$$
p_{h \mid T}=\left.\frac{\nabla r_{h} u}{\left|\nabla r_{h} u\right|}\right|_{T}, \quad \forall T \in \mathscr{T}_{h}, \quad T \subset \bar{\Omega}_{4} .
$$

On remarque par ailleurs que compte tenu de (5.4) on a

$$
|\nabla u(x)| \geqq \frac{C h}{2 \mu}, \quad \forall x \in \Omega_{4} .
$$

Il résulte de (5.30)-(5.32) que l'on peut appliquer les lemmes 5.1, avec $\delta=\mathrm{Ch} / 2 \mu$ et 5.3 , avec $\vec{p}=\nabla u$ et $\vec{q}=\nabla r_{h} u$, d'où

$$
X_{4} \leqq g\left\|\nabla\left(r_{h} u-u\right)\right\|_{\infty}^{2} \int_{\Omega_{4}} \frac{d x}{|\nabla u|+\left|\nabla r_{h} u\right|}
$$

en notant $\|\nabla v\|_{\infty}=\|\nabla v\|_{L^{\infty}(\Omega) \times L^{\infty}(\Omega)}$; on en déduit d'après $(5.20)$ et $(5.22)$ :

$$
X_{4} \leqq g \frac{C^{2}}{\mu^{2}}\left(\frac{h}{\sin \theta_{0}}\right)^{2} \int_{\Omega^{\delta}} \frac{d x}{|\nabla u|} \quad \text { avec } \delta=\frac{C h}{2 \mu},
$$

décembre 1976. 
ce qui avec (5.13) ( $c f$. lemme 5.3) implique

$$
X_{4} \leqq \frac{4 \pi}{\mu} g C\left(\frac{h}{\sin \theta_{0}}\right)^{2}\left[-h+\left(R-\frac{2 g}{C}\right)+\frac{2 g}{C} \log \left(R-\frac{2 g}{C}\right)-\frac{2 g}{C} \log h\right],
$$

soit un peu plus simplement

$$
X_{4} \leqq \frac{4 \pi}{\mu} g C\left(\frac{h}{\sin \theta_{0}}\right)^{2}\left(R-\frac{2 g}{C} \log \frac{h}{R}\right) .
$$

Compte tenu de (5.15) l'estimation (5.14) s'obtient par sommation des $X_{i}, i=1, \ldots, 6$; de façon plus précise on a, pour $h$ suffisamment petit ;

$$
\left\|u_{h}-u\right\|_{\nu} \leqq 4 \sqrt{ } \frac{-g}{\mu}\left(\frac{h}{\sin \theta_{0}}\right) \sqrt{-\log h} .
$$

\subsection{Généralisations}

$\mathrm{Au}$ vu des expériences numériques que nous avons pu effectuer, il semble (avec $f \neq$ Cte tout au moins) que dans un grand nombre de cas importants dans les applications, on ait $u \in V \cap W^{2, \infty}(\Omega)$, l'ensemble $\Omega^{0}=\{x \mid \nabla u(x)=0\}$ ayant un nombre fini de composantes connexes, compactes dans $\Omega$ et de frontière régulière; il semble par ailleurs que dans les cas ci-dessus on puisse conjecturer, si $\delta>0$, la validité de l'estimation

$$
\int_{|\nabla u(x)| \geqq \delta} \frac{d x}{|\nabla u|}=0(-\log \delta) .
$$

Dans ces conditions on démontrerait aisément, en procédant comme au paragraphe 5.3, que vaut encore l'estimation

$$
\left\|u_{h}-u\right\|_{V}=0(h \sqrt{-\log h}) .
$$

\section{CONCLUSION}

On a pu montrer que l'estimation de l'erreur d'approximation en $O(\sqrt{h})$, obtenue lorsque $f$ appartient à $L^{2}(\Omega)$, pour l'approximation de $(P)$ par une méthode d'éléments finis d'ordre un, n'est pas nécessairement optimale, puisque dans le cas particulier du domaine circulaire avec $f=\mathrm{Cte}$, une erreur en $O(h \sqrt{-\log h})$ a pu être obtenue. A partir de ce cas particulier, des propriétés de la solution de $(P)$ assurant le même ordre de convergence ont pu être dégagées.

On notera par ailleurs que dans la démonstration du théorème 5.1 donnant les estimations d'ordre « quasi optimal » de l'erreur d'approximation, il a été fait usage du multiplicateur $p$ dont l'existence résulte du théorème 3.1. 
Il semble, d'une façon générale, que l'emploi des multiplicateurs peut faciliter l'étude de l'approximation des inéquations variationnelles, moyennant probablement la généralisation des résultats de Brezzi [2] sur l'approximation de certains problèmes de points-selle puisqu'en fait ( $c f$. remarque 3.2 ) le couple $\{u, p\}$ mis en évidence au théorème 3.1 est point-selle sur $V \times \Lambda$ de la fonctionnelle $\mathscr{L}$ définie par

$$
\mathscr{L}(v, q)=\frac{\mu}{2} \int_{\Omega}|\nabla v|^{2} d x+g \int_{\Omega} q . \nabla v d x-\langle f, v\rangle .
$$

\section{BIBLIOGRAPHIE}

1 H. Brezis, Monotonicity in Hilbert Spaces and some Applications to Non Linear Partial Differential Equations, dans Contributions to Non Linear Functional Analysis, E. Zavantonello ed., Acad. Press, New York, 1971, p. 101-156.

2. F. BrezzI, On the Existence, Uniqueness and Approximation of Saddle-Point Problems Arising from Lagrangian Multipliers, R.A.I.R.O., R 2, août 1974, p. $129-151$.

3. O. BristeaU, Application de la méthode des éléments finis à la résolution numérique d'inéquations variationnelles d'évolution de type Bingham, Thèse de $3^{\mathrm{e}}$ cycle, Université Paris VI, 1975.

4. O. Bristeau et R. Glowinski, Finite Element Analysis of the Unsteady Flow of a Viscous-Plastic Fluid in a Cylindrical Pipe, dans Finite Element Methods in Flow Problems, Oden, Zienkiewicz, Gallagher, Taylor ed., University of Alabama Press, Huntsville, 1974, p. 471-488.

5. J. CEA et R. GLOwINSKI, Méthode numérique pour l'écoulement laminaire d'un fluide rigide viscoplastique incompressible, Inter. J. Computer Math., sect. B, vol. 3, 1972, p. 225-255.

6. P.-G. Ciarlet et P.-A. Raviart, General Lagrange and Hermite Interpolation in $R^{n}$ with Applications to Finite Element Methods, Arch. Rat. Mech. Anal., vol. 46, 1972, p. 177-199.

7. P.-G. Ciarlet et C. Wagschal, Multipoint Taylor Formulas and Applications to the Finite Element Methods, Numer. Math., vol. 17, 1971, p. 84-100.

8. G. Duvaut et J.-L. Lions, Les inéquations en mécanique et en physique, Dunod, Paris 1972.

9. I. Ekeland et R. Temam, Analyse convexe et problèmes variationnels, Dunod et Gauthier-Villars, Paris, 1974.

10. M. Fortin, Calcul numérique des écoulements des fluides de Bingham et des fluides newtoniens incompressibles par des méthodes d'éléments finis, Thèse, Université Paris VI, 1972.

11. P. Germarn, Mécanique des milieux continus, Masson, Paris, 1962.

12. R. Glowinski, J.-L. Lions et R. TrÉmolières, Analyse numérique des inéquations variationnelles, Dunod, Paris, 1976.

13. J.-L. Lions, Contrôle optimal des systèmes gouvernés par des équations aux dérivées partielles, Dunod et Gauthier-Villars, Paris, 1968.

14. P. P. Mosolov et V.P. Miasnikov, Variational Methods in the Theory of the Fluidity of a Viscous-Plastic Medium, J. Mech. and Appl. Math. (P.M.M.), vol.. 29, no 3, 1965, p. 46-492.

décembre 1976. 
15. P. P. Mosolov et V. P. Miasnikov, On Stagnant Flow Regions of a Viscous Plastic Medium in Pipes, J. Mech. and Appl. Math. (P.M.M.), vol. 30, $\mathrm{n}^{\circ} 4$, 1966, p. 705-719.

16. P. P. Mosolov et V. P. Miasnikov, On Qualitative Singularities of the Flow of a Viscous Plastic Medium in Pipes, J. Mech. and Appl. Math. (P.M.M.), vol. 31, $\mathrm{n}^{\circ} 3,1967$, p. 581-585.

17. J. NeCAs, Les méthodes directes en théorie des équations elliptiques, Masson, Paris, 1967.

18. W. Prager, Introduction to Mechanics of Continua, Ginn and Company, 1961.

19. Strang et G. Fix, An Analysis of the Finite Element Method, Prentice Hall, New York, 1973. 